\title{
Segmentación de los consumidores de alimentos orgánicos según sus actitudes, valores y creencias ambientales
}

\author{
Segmentation of organic food consumers based on their attitudes, values and \\ environmental beliefs \\ Lizbeth Salgado Beltrán* \\ Universidad de Sonora, México
}

Recibido el 7 de mayo de 2017; aceptado el 4 de abril de 2018

Disponible en Internet el: 5 de noviembre de 2018

\section{Resumen}

El objetivo de este trabajo fue segmentar a los consumidores según sus actitudes, valores y creencias ambientales en la decisión de compra de productos orgánicos en la zona norte de Sonora, para proporcionar recomendaciones a los actores (organizaciones públicas y privadas) que intervienen en el mercado de alimentos orgánicos. Para ello, se hizo inicialmente un Análisis Factorial Exploratorio (AFE) y después un análisis clúster para identificar los segmentos de los consumidores por sus actitudes, valores y creencias, se identificaron dos segmentos (participativo ambientalmente y consistente ambientalmente). Este hallazgo presenta oportunidades y retos para las organizaciones privadas y públicas, para las primeras, al considerar las agrupaciones de los consumidores, podrán diseñar estrategias de marketing sobre los productos orgánicos y para las segundas, deben incluir la educación ambiental en sus estímulos para mejorar la concientización de las personas.

Códigos JEL: M310, Q100

Palabras clave: Marketing; Actitudes; Consumidores; Creencias; Valores.

\footnotetext{
*Autor para correspondencia

Correo electrónico lizbeth.salgado@unison.mx (L. Salgado Beltrán)

La revisión por pares es responsabilidad de la Universidad Nacional Autónoma de México
} 


\begin{abstract}
The objective of this work was to segment consumers based on their attitudes, values and environmental beliefs in the purchase decision of organic products in the northern zone of Sonora, to provide recommendations to the actors (public and private organizations) that involved in the organic food market. To do this, an Exploratory Factor Analysis (AFE) was initially carried out and then a cluster analysis to segment the consumers by their attitudes, values and beliefs. Two segments were identified (environmentally participative and environmentally consistent). This finding presents opportunities and challenges for private and public organizations, for the first considering consumer segments in the area, they may devise marketing strategies on organic products and for the second ones, they must include environmental education in their stimuli to improve awareness of people.
\end{abstract}

JEL codes: C38, M31, Q13,

Keywords: Marketing; Attitudes; Consumers; Beliefs; Values.

\title{
Introducción
}

En las últimas décadas la temática ambiental ha tomado fuerza entre las organizaciones incentivando el desarrollo de procesos de producción que contribuyen a la sustentabilidad de la economía y la sociedad (Salgado et al., 2006). Este escenario ha sido originado primero, por el incremento de la preocupación de las personas por el rápido deterioro del medio ambiente por la acción humana (Fraj, 2003) y segundo, la aceptación generalizada del concepto de desarrollo sustentable como aquel que satisface las necesidades de las generaciones presentes sin comprometer la capacidad de las futuras generaciones para satisfacer sus propias necesidades (Brundtland, 1987). El desarrollo sustentable recae en tres pilares: calidad ambiental, justicia social y prosperidad económica, que están interconectados (Elkington, 1997). Desde una perspectiva del marketing, se han considerado las 3 P's (beneficio, personas y planeta) (Placet et al., 2005) que se retroalimentan entre sí y se deben cumplir simultáneamente (Balderjahn et al., 2013).

En este contexto, la agricultura orgánica es una alternativa de producción que conjuga objetivos ambientales, sociales y económicos (Romero Valenzuela, et al., 2016). Tal es la relevancia que al año 2015, 179 países del mundo tenían actividades orgánicas con un total de 43,7 millones de hectáreas; cerca de la mitad de esa extensión se encuentra en Oceanía, una cuarta parte en Europa y un poco menos en América Latina, Australia es el país con la mayor cantidad de área bajo manejo orgánico (7,6 millones ha), dentro de Europa esta España ( 2 millones ha) y en América Latina esta Argentina ( 3 millones ha) (IICA, 2016, IFOAM, 2016). En el caso de México cuenta con 200,039 productores (FIBL, 2017), de las cuales 88,3 están en proceso de certificación (SAGARPA, 2016). Particularmente, el Estado de Sonora tiene una superficie orgánica entre 1972 a 3140 hectáreas, divida en 7 municipios (SAGARPA, SIAP, 2016), se encuentra dentro de los 4 principales estados productores de carne orgánica (SAGARPA, SIAP, 2017) con una tendencia creciente, además, ha desarrollado la innovación en alimentos básicos como el trigo orgánico. Si bien no se presentan datos sobre el consumo orgánico a nivel estatal, la demanda nacional crece en un 10 por ciento anual (Ramírez, 2016), 
la venta nacional en tiendas de autoservicio se ha incrementado en un 20 por ciento, en tiendas especializadas en un 10 por ciento (SAGARPA, 2013) y el incremento de conductas ambientales según el índice Greendex de National Geographic (2014) pasó de 53.9 (2012) a 55 (2014) en una escala de 41-65 puntos. De forma inversa, aquellos países más productores de alimentos orgánicos tienen niveles de conductas ambientales menores a México, por ejemplo, España (51.3) y Australia (50.4). Con ello, se refleja la importancia progresiva del mercado orgánico en México y la oportunidad de los productores sonorenses de hacer negocios en los mercados externos e internos.

Desde una óptica del comportamiento del consumidor, existen dos situaciones, por un lado, la homogeneización y por el otro, el consumo diferenciado (alimentos orgánicos) dirigido a nuevos segmentos de consumidores (Gutiérrez et al., 2012). En este sentido, los estudios sobre el comportamiento del consumidor ambiental han sido abordados desde características sociodemográfica. Sin embargo, Schlegelmilch et al., (1994), tras un análisis exhaustivo señalan que en el caso de las variables sociodemográficas explican menos del 10 por ciento de la variación del comportamiento ecológico. Es decir, por sí solas no pueden generar una compra de alimentos orgánicos, sino por otras condiciones como la disponibilidad del producto, ventas, entre otros, donde el efecto debiera ser directo (Orduña et al., 2009). Aun así, se han elaborado perfiles basados solo con estas variables (Wier y Calverley, 2002) que han impactado en el desarrollo de estrategias para posicionar este tipo de productos. Respecto a perfiles psicográficos, se han basado en los valores (Peattie 2001; McCarty y Shrum 1994; Stern et al., 1993), las creencias (Crane 2000; Chan 1999; Vining y Ebreo 1990; Alba y Hutchinson 1987), las actitudes (Dietz et al., 1998; Schlegelmilch et al., 1994) y los estilos de vida (Fraj et al., 2004) como variables influenciadoras de conductas ambientales.

Los estudios sobre comportamientos ambientales del consumidor mexicano, incluida la compra de productos orgánicos, son incipientes, y se han centrado en: conductas protectoras del ambiente (Corral, 1996; Corral-Verdugo \& Armendáriz, 2000; Corral et al., 2009) y los motivadores e inhibidores de la compra ecológica (Salgado y Beltrán, 2011; Aguilar Salinas et al., 2012; Gutiérrez et al., 2012; Carrete et al., 2012; Salgado y Bravo, 2015; Díaz Víquez, et al., 2015; González et al, 2015; Escobar-López et al., 2017), la contribución de este trabajo va en esta última área, al profundizar en la aplicación de las escalas del comportamiento donde las actitudes, valores y creencias del consumidor han sido las variables ambientales mayormente estudiadas (Ramsey y Rickson, 1976; Alba y Hutchinson 1987; Vining y Ebreo 1990; Stern et al., 1993; Schlegelmilch et al.,1994; McCarty y Shrum 1994; Dietz et al., 1998; Chan 1999; Bechtel et al., 1999; Crane 2000; Peattie 2001; Diamantopoulos et al., 2003; Balderjahn et al., 2013) y no se ha encontrado consenso sobre la influencia de estas variables en la compra de alimentos orgánicos al concurrir diversas contradicciones (Grubor y Nenad, 2016), siendo necesario ampliar en este sentido y segundo, permite identificar los segmentos para un mayor entendimiento del consumidor de productos orgánicos para la diferenciación de las estrategias de marketing por parte de los productores de la región estudiada.

De ahí que el objetivo de este trabajo fue segmentar a los consumidores según sus actitudes, valores y creencias ambientales en la decisión de compra de productos orgánicos en la zona norte de Sonora, para proporcionar recomendaciones a los actores (organizaciones públicos y privados) que intervienen en el mercado de alimentos orgánicos. 


\section{Revisión de literatura}

En Marketing, los patrones de conducta han recibido atención porque frecuentemente sirven como bases para la segmentación del mercado (Holt, 1997). El concepto de segmentación del mercado se introdujo como una alternativa para la diferenciación del producto (Nair y Berthon, 2003), sin embargo, las variables socioeconómicas y demográficas han perdido capacidad de diferenciación y de ventaja comparativa, especialmente porque la segmentación derivada de su utilización tiende a homogeneizar las estrategias empresariales (Rivas y Grande, 2004), dando mayor ventaja al uso de la segmentación psicográfica. En el aspecto ambiental, para los autores Corral et al., (2009); Bratt (1999); Corral (1996); Lee et al., (1995) no existe una categoría general de "conducta proecológica" (CPE), sino que la misma se manifiesta como un conjunto de factores múltiples independientes, aunque existe evidencia de que pudieran relacionarse entre sí (Diamantopoulos et al., 2003; Tracy y Oskamp 1984). De tal forma, que aparece una estructura de factores diversos que influyen en la adquisición de alimentos orgánicos que es necesario analizar, dentro de los cuales, los más estudiados en una son las actitudes, los valores, las creencias (Ramsey y Rickson, 1976; Alba y Hutchinson 1987; Vining y Ebreo 1990; Stern et al., 1993; Schlegelmilch et al.,1994; McCarty y Shrum 1994; Dietz et al., 1998; Chan 1999; Bechtel et al., 1999; Crane 2000; Peattie 2001; Diamantopoulos et al., 2003; Balderjahn et al., 2013).

\section{Actitudes, valores y creencias}

Uno de los componentes es la actitud, definida como la predisposición a evaluar un símbolo, un objeto, una acción, un producto o marca de manera favorable o positiva o, por el contrario, desfavorable o negativa (Shiffman y Lazar, 2010). Se caracteriza por su dificultad de medición, por su rigidez de cambio cuando ya están formadas y por la creación de estereotipos (Fraj et al., 2004). La actitud es un indicativo de conducta no la conducta en sí.

La actitud ecológica ha sido estudiada a través del tiempo como una variable importante en la decisión de compra ecológica, uno de los primeros estudios (Maloney y Ward, 1973) al respecto lo hace enfocándose desde la perspectiva de la psicología. En los aspectos actitudinales propios de la conducta ambiental, las primeras variables utilizadas que caracterizaron Maloney y Ward (1973) fueron el compromiso verbal y real de participación en actividades pro ambientales, el efecto y conocimiento de los problemas ambientales.

Algunos estudios posteriores (Ahmed et al., 2001; Chan, 2001; Laroche et al., 2001; Kotchen y Reiling, 2000) han relacionado la actitud con variables sociodemográficas, tal es el caso de que las personas con un nivel alto de ingresos tienen más actitudes hacia la compra de alimentos orgánicos. Partiendo del planteamiento de que a medida que el ingreso de los consumidores aumenta, la demanda se traslada hacia productos que otorgan conveniencia y satisfacen atributos de calidad y son las actitudes las que juegan un rol preponderante para comprender las preferencias de los consumidores (Rodríguez, et al., 2002). Así como significancias vinculadas a los aspectos afectivos de la actitud y la compra de estos alimentos. Es así que, las actitudes positivas hacia el medio ambiente no son necesariamente indicativo de altos niveles de conocimiento ambiental (Diamantopoulos et al., 2003; Bagozzi et al., 1992; Ramsey y Rickson, 1976), sin embargo, las actitudes hacia los alimentos orgánicos tienen un 
positivo efecto en las intenciones de compra (Michaelidou y Hassan, 2008; Kim y Chung, 2011; Pino et al., 2012; Hsu et al., 2016).

Otros aspectos que influyen significativamente en el comportamiento de las personas, son los valores (Beatty et al., 1985; Yankelovich, 1981; Williams, 1979; Rokeach, 1973). A diferencia de otros elementos, los valores se van aprendiendo durante los procesos de socialización, en los cuales los individuos siguen normas colectivas que les garantizan la integración en un grupo y la normalización de su personalidad (González, 2000). Schwartz (1994) define a los valores humanos como objetivos deseables, que varían en importancia, que sirven como principios rectores en las vidas de las personas. Es por ello que se considera que las personas no nacen con valores, sino que los aprenden a través del contacto con otras personas (Rose y Shoham, 2000; Kahle y Goff, 1983). Los valores personales proporcionan información de las aspiraciones y metas que las personas evalúan como más importantes, de ahí que permitan conocer y determinar las motivaciones que influyen en sus decisiones de consumo (Kahle et al., 2000). De tal forma que, los valores son fundamentales para la cultura y ejercen una fuerte influencia en la recepción y la percepción de los símbolos y mensajes incrustados en la publicidad (Watson et $a l ., 2002)$, son una influencia significativa en las actitudes y comportamientos (Ip, 2003). Por lo tanto, los valores son creencias subjetivas y emocionales (Smith y Schwartz, 1997). En el caso de los consumidores con valores ecológicos más fuertes (aquellos que pueden hacer posible un cambio), tienden a tomar decisiones consistentes con el consumo sustentable (Haws et al., 2014). Los autores Thøgersen y Olander (2002) al analizar los valores con comportamiento ecológico, encontraron que se requiere que los individuos restrinjan las tendencias egoístas. Por otro lado, Kim (2011) descubrió una influencia positiva del colectivismo sobre el consumidor ecológico hacia los valores auto trascendentales. Sin embargo, ni el género ni la escala de valores presenta un impacto significativo respecto al uso del papel, por ejemplo, según estudio de Catlin y Wang (2013).

La modificación de las creencias sobre el medio ambiente en la sociedad pudo haberse iniciado con la revelación científica de los efectos nocivos de la degradación del medio ambiente (Heberlein, 1972) por ejemplo en la salud, como es el uso de pesticidas en productos alimenticios, fiebre porcina y gripe aviar, entre otros (Mesías Díaz, et al., 2012). Estas creencias forman parte del conocimiento y las valoraciones que le da cada individuo acerca de algo (Ellen, 1994). Autores como Schwartz (1970; 1977) en su modelo de ActivaciónNorma analiza el comportamiento como una función de creencias sobre las consecuencias de acciones y normas sobre la responsabilidad personal para llevar a cabo acciones específicas en respuesta. Catton y Dunlap (1978); Dunlap y Van Liere (1978) desarrollan un nuevo modelo sobre las relaciones entre el ser humano y el medio ambiente denominado "Nuevo Paradigma Ambiental o Ecológico (NEP por sus siglas en inglés), se centra en las creencias sobre las habilidades humanas para alterar el equilibrio de la naturaleza, sobre la existencia de límites en el crecimiento de las sociedades humanas y sobre el derecho de los seres humanos a gobernar la Naturaleza. La Escala NEP de Dunlap y Van Liere (1978) ha llegado a ser la medida más usada para la orientación ecológica. Más tarde surge la teoría de los Valores-Creencias-Normas (VBN por sus siglas en inglés) hacia el medio ambiente planteado por Stern et al., (1993) y Stern (2000), mide las creencias asociadas a las consecuencias que pueden tener las condiciones medio ambientales para los otros, para uno mismo o para la biosfera en su conjunto. Encuentran que estas creencias son más fuertes en las mujeres que los hombres. Por lo tanto, las creencias de los consumidores son que ellos como individuos pueden ayudar a solucionar los problemas 
ambientales (Roberts, 1996). Las creencias, por tanto, han cobrado un papel determinante para entender la relación del ser humano y el medio ambiente.

\section{Metodología}

\section{Instrumento de medición}

Para cumplir con el objetivo de segmentar a los consumidores según sus actitudes, valores y creencias ambientales en la decisión de compra de productos orgánicos en la zona norte de Sonora, se revisó ampliamente la literatura y se utilizaron 3 escalas: 1) Actitudes (Maloney et al., 1975; 2) Valores (Haws et al., 2014); y 3 ) Creencias (Dunlap et al., 2000).

El instrumento de medición está compuesto de 3 apartados: el primero contiene los hábitos de consumo de productos orgánicos, el segundo se compone de las 3 escalas antes descritas, y en el tercero, se incluyeron variables sociodemográficas como edad, nivel de ingresos y país de residencia, esta última variable solo se utilizó para filtrar a los individuos en la frontera de Nogales, México y Nogales, Arizona. Para asegurar de que los consumidores estuviesen entendiendo lo que se les estaba preguntando, se incluyó la leyenda: "Producto orgánico: es aquel producto libre de pesticidas y que en todo su proceso de producción su impacto al medio ambiente es menor".

La escala revisada de actitudes y conocimiento medioambiental (EAKS) desarrollada por Maloney et al., (1975) mide la actitud a través de los 3 niveles de compromiso ambiental (Consiste en 3 preguntas compuestas de 10 ítems cada una): 1) compromiso verbal, mide el elemento intencional; 2) compromiso real, mide el elemento de comportamiento y, 3) compromiso afectivo, mide el elemento afectivo (Tabla 1). Dado que las 3 escalas fueron originalmente redactadas en inglés, se hizo la traducción de los ítems para posteriormente hacer una prueba piloto (pre-stest) con 25 personas, en la cual se ajustaron algunos ítems para su mejor comprensión. Las respuestas se puntearon en una escala Likert de 5 puntos.

Tabla 1. Items de la Escala EAKS.

Compromiso Verbal

1.Estaría dispuesto a llevar una bici o a coger el autobús para ir a trabajar con el fin de reducir la contaminación del aire.

2.Probablemente, nunca me uniría a un grupo el cual estuviese únicamente preocupado por los principios medioambientales (I)

3. Estaría dispuesto a usar un sistema de tránsito rápido para ayudar a reducir la contaminación del aire.

4. No estaría dispuesto a dejar de conducir durante un fin de semana por una alerta de humo (I)

5. No estoy dispuesto a salirme de mi manera de hacer las cosas por motivos ecológicos ya que se trata de un asunto del Gobierno (I).

6. Donaría la paga de un día a una fundación para ayudar a mejorar el medio ambiente

7. Pararía de comprar productos de empresas que contaminan el medio ambiente, incluso aunque fuese un inconveniente para mí.

8. Escribiría a un semanario relacionado con los problemas medioambientales.

9. Probablemente no iría de casa en casa a distribuir publicidad e información sobre el medio ambiente (I) 
10. No pagaría un impuesto medioambiental por la contaminación aunque ello supusiese una disminución del problema de la polución (I).

Compromiso Real

11. Nunca he comprado un producto porque tuviera menores efectos contaminantes (I)

12. Sigo al tanto de mi diputado al que vote sobre los principios medioambientales.

13. Nunca he escrito a un diputado en relación con los problemas medioambientales (I)

14. He contactado con el departamento de Medio Ambiente de mi Comunidad Autónoma o Ayuntamiento para informarme sobre lo que puedo hacer para reducir la contaminación.

15. No hago ningún esfuerzo especial para comprar productos con embalajes reciclables (I)

16. He asistido a un seminario ofrecido por una organización especialmente preocupada por mejora el medio ambiente.

17. He cambiado de productos por razones ecológicas.

18. Nunca he participado en un acto que se preocupara por aspectos medioambientales (plantar un árbol, limpieza de parques, etc.). (I)

19. Nunca he ido a una conferencia sobre ecología (I)

20. Me he suscrito a publicaciones ecológicas.

Compromiso Afectivo

21. Siento que la gente se preocupa demasiado por la existencia de pesticidas en los productos alimenticios.

22. Me asusta pensar que la comida que ingiero está contaminada con pesticidas.

23. Me enfurece pensar que el Gobierno no hace nada para ayudar a controlar la contaminación del medio ambiente.

24. Me es indiferente lo que dice la siguiente sentencia: "El mundo morirá en 40 años como no demos más importancia al medio ambiente (I)

25. Llego a indignarme cuando pienso sobre el daño causado a las plantas y vida animal por la contaminación.

26. Normalmente no me preocupa la "contaminación acústica" (I)

27. Me deprimo en los días que hay contaminación en el ambiente (humos, malos olores).

28. Cuando pienso en como contaminan las industrias, me enojo y frustro.

29. Nunca me ha afectado la contaminación ya que se exagera mucho sobre el tema (I)

30. Casi nunca me preocupo sobre los efectos que el humo puede causar en mi familia y en mí.

Nota: $(I)$ = Las puntuaciones de estos ítems se invierten. Los ítems en cursiva se eliminaron después del análisis de consistencia interna.

Fuente: Maloney et al. (1975).

Se ha incorporado la escala GREEN (Haws et al., 2014) para medir los valores ambientales de los consumidores. Los consumidores con valores más fuertes tenderán a tomar decisiones consistentes con el consumo sustentable. Se mide en 6 ítems. Las respuestas se puntearon en una escala Likert de 5 puntos (Tabla 2). 
Tabla 2. Ítems de la Escala GREEN.

1. Es importante para mi que los productos que uso no dañen el medio ambiente.

2. Considero el impacto potencial al medio ambiente de mis acciones al tomar la mayoría de mis decisiones.

3. Mis hábitos de compra son afectados por mi preocupación por nuestro medio ambiente.

4. Estoy preocupado sobre el desperdicio de los recursos de nuestro planeta.

5. Me describiría a mi mismo como responsable ambientalmente.

6. Estoy dispuesto a ser molestado con el fin de tomar acciones que son más amigables con el medio ambiente.

Fuente: Haws et al (2014).

Para medir las creencias se utilizó la escala del Nuevo Paradigma Ecológico (NEP) desarrollada por Dunlap y Van Liere (1978) y actualizada en el 2000 (Tabla 3). Originariamente, el instrumento creado para recoger estas creencias generales incluía un total de 12 ítems, pero en la última revisión de la escala se incluyeron tres ítems más (Dunlap et al., 2000).

Tabla 3. Escala NEP.

Nos estamos acercando al límite del número de personas que la tierra puede soportar

Los seres humanos tienen el derecho a modificar el entorno natural para adaptarlo sus necesidades

3. Cuando los humanos interfieren con la naturaleza a menudo tienen desastrosas consecuencias

4. El ingenio humano asegurará de que NO hagamos de la tierra un lugar inhabitable

Los seres humanos están abusando gravemente del medio ambiente.

La tierra tiene muchos recursos naturales, si sólo aprendemos cómo desarrollarlos

Las plantas y los animales tienen el mismo derecho que los seres humanos a existir

El equilibrio de la naturaleza es suficientemente fuerte para hacer frente a los impactos de las naciones industriales modernas

A pesar de nuestras habilidades, los seres humanos todavía están sujetos a las leyes de la naturaleza

La llamada "crisis ecológica" que enfrenta la humanidad ha sido en gran medida exagerada

La tierra es como una nave espacial con ambiente y recursos muy limitados

Los seres humanos estaban destinados a gobernar sobre el resto de la naturaleza

El equilibrio de la naturaleza es muy delicado y fácilmente de alterarse

Los seres humanos finalmente aprenderán lo suficiente acerca de cómo funciona la naturaleza para poder controlarla

Si las cosas siguen su curso, pronto experimentará un catástrofe ecológica

Nota: Los ítems en cursiva se eliminaron después del análisis de consistencia interna.

Fuente: Dunlap et al. (2000)

\section{Participantes}

El estudio se llevó a cabo entre consumidores de la zona norte de Sonora, se seleccionaron los entrevistados mayores de edad, que han consumido alimentos orgánicos en los últimos 6 meses, por medio de un muestreo por conveniencia se les aplicó la encuesta personal (face 
to face), en los principales centros comerciales de la zona norte de Sonora. El muestreo por conveniencia es usado en estudios exploratorios para poder tener una aproximación del objeto de estudio (Grande y Abascal, 2014). Para el cálculo de la muestra se tomó como referencia los datos del Instituto Nacional de Estadística y Geografía (INEGI) y la formula de población infinita (Tabla 4).

Tabla 4. Ficha técnica.

\begin{tabular}{ll}
\hline Universo & Consumidores de la zona norte de Sonora \\
Nivel de confianza & $95 \%$ \\
Margen de posible de error & de $+4.36 \%$ \\
Muestra & 506 \\
Muestreo & Por conveniencia \\
Fecha de recogida de datos & Enero-marzo del 2015 \\
\hline
\end{tabular}

Fuente: Elaboración propia

Análisis de los datos

Inicialmente se calcularon estadísticos univariantes en el paquete estadísticos SPSS versión 20.0, con el objetivo de estudiar el comportamiento de las variables en forma individual al tener una primera impresión de la tendencia de los resultados (Pedret et al., 2003). Para aumentar la exactitud de la medida se realizó el análisis de consistencia interna por medio del alfa de Cronbach. Se realizaron varios análisis factoriales de las escalas, por método de extracción de análisis de componentes principales con rotación Varimax, aplicándose la medida de adecuación muestral de Kaiser-Meyer-Olkin (KMO) y la prueba de esfericidad de Bartlett con la finalidad de depurar las escalas y agrupar los ítems en varios factores. Para poder identificar los grupos de consumidores, se llevó a cabo un análisis clúster o de conglomerados en dos fases, primero, se hizo un análisis clúster jerárquico para detectar los segmentos por medio del criterio de Ward (1963), el cual crea grupos minimizando la varianza intra-grupo y para cada grupo que resultaría de la fusión de dos grupos determinados, calcula su centro de gravedad, es decir la media del grupo en cada variable. Segundo, se realizó un análisis cluster de k-medias con el objetivo de afinar los segmentos por actitudes, valores y creencias y conocer sus características.

\section{Resultados}

\section{Análisis sociodemográfico y hábitos de consumo}

Sobre los resultados sociodemográficos, la muestra se concentra en personas relativamente jóvenes, el 39.1 por ciento tiene entre 25-35 años, seguido del grupo de 18-24 años con un 21.7 por ciento, el 19.6 por ciento tiene entre 36-44 años, el 13 por ciento entre 45-54 años, de 55-64 años en adelante son el grupo minoritario (6.5 por ciento). Respecto a los niveles de ingreso familiar, el 67.4 por ciento cuenta con un nivel de ingreso familiar medio, el 19.6 por ciento medio alto, el 8.7 por ciento medio bajo y los niveles alto y bajo 2.2 por ciento respectivamente. Sobre los hábitos de consumo, el 76.1 por ciento ha consumido productos orgánicos en los últimos 6 meses, de los cuales el 45.5 por ciento los ha consumido con una frecuencia de una 
vez al mes, el 22.7 por ciento diariamente, el 18.2 por ciento una vez a la semana y el resto (13.6 por ciento) una vez cada 15 días. El principal lugar de compra de los productos orgánicos son los supermercados (41.3 por ciento), seguido por las tiendas especializadas (19.6 por ciento), de mi huerta (10.9 por ciento) y en menor medida tienda pequeña del barrio y al productor con 8.7 por ciento respectivamente, frutería con un 4.3 por ciento y venta por internet con 2.2 por ciento (Tabla 5).

Los productos orgánicos más consumidos en la zona norte de Sonora son aquellos sin procesar o con bajo procesamiento, en primer lugar, están las verduras con un 63 por ciento, seguido de las frutas con un 52.2 por ciento, el 30.4 por ciento corresponde a pan, cereales, arroz y pastas, leche, yogurt y queso con un 26.1 por ciento, el 19.6 por ciento consumen grasas, aceites y dulces y en último lugar, las carnes, aves, legumbres, frutos secos y huevos con un 8.7 por ciento (Tabla 6).

Tabla 5. Frecuencia y lugar de adquisición de productos orgánicos de la zona norte de Sonora.

\begin{tabular}{lll}
\hline En los últimos 6 meses ha consumido productos orgánicos & Si & No \\
\hline
\end{tabular}

Frecuencia

Diariamente 22.7

Una vez a la semana 18.2

Una vez cada 15 días 13.6

Una vez al mes 45.5

Lugar de adquisición

Supermercado 41.3

Tienda especializada 19.6

De mi huerto 10.9

Tienda pequeña del barrio

Al productor

Frutería

Venta por internet 2.2

Fuente: Elaboración propia 
Tabla 6.

Alimentos orgánicos más consumidos en la zona norte de Sonora.

\begin{tabular}{ll}
\hline Productos orgánicos & Porcentaje \\
\hline Verduras & $63.00 \%$ \\
Frutas & $52.20 \%$ \\
Pan, cereales, arroz y pastas & $30.40 \%$ \\
Leche, yogurt y queso & $26.10 \%$ \\
Grasas, aceites y dulces & $19.60 \%$ \\
Carnes, aves, legumbres, frutos secos y huevos & $8.70 \%$ \\
\hline
\end{tabular}

Fuente: Elaboración propia

\section{Análisis de consistencia}

Se llevó a cabo el análisis de consistencia interna, los resultados del alfa de Cronbach de las subescalas de EAKS presentan coeficientes muy modestos: compromiso verbal $\alpha=.675$; real $\alpha=.609$ y afectivo $\alpha=.631$, se prescindió de aquellos ítems que no se relacionaban bien con el resto de la escala, es decir se consideraron aquellos ítems con mayor consistencia interna (> a 60 por ciento) en el caso de estudios exploratorios (Miquel et al., 1996) así de los 30 ítems se eliminaron 14, quedando reducida la escala final a 16 ítems (Tabla 7). Las cifras de los factores $(\alpha=.675 ; \alpha=.609$ y $\alpha=.631)$ son similares a las conseguidas en otros estudios (Fraj et al., 2004; Fraj y Martínez 2005; Nisbet et al., 2009). En el caso de la escala GREEN se obtuvo un coeficiente de $\alpha=0.773$ similar a la investigación de Bhatia y Jain (2013). La consistencia interna es muy buena y no fue necesario eliminar ítems. Respecto a la escala NEP, de los 15 ítems, se eliminaron 4, con los siguientes valores: la realidad de los límites del crecimiento $\alpha=.690$; posibilidad de una ecocrisis $\alpha=.586$; rechazo a la excepción $\alpha=.526$ y la fragilidad del balance de la naturaleza $\alpha=.641$. La escala presenta de bajos a moderados coeficientes de consistencia interna.

\section{Análisis factorial}

Se realizó un análisis factorial exploratorio, para la escala EAKS que mide las actitudes en 3 dimensiones: compromiso verbal, compromiso real y compromiso afectivo, se reagruparon en 7 factores que explican un 78.04 por ciento de la varianza presentando un porcentaje adecuado de acuerdo con la sugerencia de Hair et al., (2005) y Del Barrio y Luque (2000), el test de esfericidad de Bartlett fue significativo y la medida de adecuación muestral de KaiserMeyer-Olkin (KMO) obtuvo el valor de 0.733, comprobando que existe una correlación entre las variables para llevar a cabo el análisis factorial en la muestra de consumidores. Así el compromiso verbal está compuesto por 3 factores: F1 compromiso intencional (3 ítems), F4 de colaboración ambiental ( 2 ítems) y F7 cambio en los estilos de vida ( 2 ítems); el compromiso real fue la dimensión que más ítems se eliminaron quedando 1 factor, F6 participación activa 
ambiental ( 2 ítems) y por el contrario, en la dimensión afectiva solo se eliminaron dos ítems, está compuesta por 3 factores: F2 interés por el medio ambiente (3 ítems), F3 frustración por las acciones de organizaciones ( 2 ítems) y F5 preocupación por la contaminación (2 ítems).

La escala GREEN está formada por 2 factores: F1 conservación ambiental (3 ítems) y F2 respeto al medio ambiente (3 ítems) que explican un 71.77 por ciento de la varianza, el test de esfericidad de Bartlett fue significativo y la medida de adecuación muestral de Kaiser-MeyerOlkin (KMO) obtuvo el valor de 0.731.

Tabla 7. Análisis factorial.

\begin{tabular}{|c|c|c|c|c|c|}
\hline Actitudes & Varianza & Valores & Varianza & Creencias & Varianza \\
\hline $\begin{array}{l}\text { F1. Compromiso } \\
\text { intencional }\end{array}$ & $19.74 \%$ & $\begin{array}{l}\text { F1. Conservación } \\
\text { ambiental }\end{array}$ & $49.45 \%$ & $\begin{array}{l}\text { F1. La realidad } \\
\text { de los límites del } 2 \\
\text { crecimiento }\end{array}$ & $22.09 \%$ \\
\hline $\begin{array}{l}\text { F2. Interés por el } \\
\text { medio ambiente }\end{array}$ & $16.28 \%$ & $\begin{array}{l}\text { F2. Respeto al medio } \\
\text { ambiente }\end{array}$ & $22.31 \%$ & $\begin{array}{l}\text { F2. Posibilidad de } \\
\text { una ecocrisis }\end{array}$ & $17.17 \%$ \\
\hline $\begin{array}{l}\text { F3. Frustración } \\
\text { por las acciones de } \\
\text { organizaciones }\end{array}$ & $11.80 \%$ & & & $\begin{array}{l}\begin{array}{l}\text { F3. Rechazo a la } \\
\text { excepción }\end{array} \\
\text { la }\end{array}$ & $14.56 \%$ \\
\hline $\begin{array}{l}\text { F4. Compromiso } \\
\text { de colaboración } \\
\text { ambiental }\end{array}$ & $9.52 \%$ & & & $\begin{array}{l}\text { F4. La fragilidad } \\
\text { del balance de la } \\
\text { naturaleza }\end{array}$ & $11.76 \%$ \\
\hline $\begin{array}{l}\text { F5. Preocupación por } \\
\text { la contaminación }\end{array}$ & $7.53 \% \%$ & & & & \\
\hline $\begin{array}{l}\text { F6. Participación } \\
\text { activa ambiental }\end{array}$ & $6.87 \%$ & & & & \\
\hline $\begin{array}{l}\text { F7. Cambio en estilos } \\
\text { de vida }\end{array}$ & $6.28 \%$ & & & & \\
\hline Total Varianza: $78.04 \%$ & & Total Varianza: $71.77 \%$ & & Total Varianza: $65.58 \%$ & \\
\hline $\begin{array}{l}\text { Medida de adecuación } \\
\text { muestral de Kaiser- } \\
\text { Meyer-Olkin (KMO): }\end{array}$ & 0.733 & 0.731 & & 0.679 & \\
\hline $\begin{array}{l}\text { Prueba de esfericidad } \\
\text { de Bartlett: }\end{array}$ & $\begin{array}{l}92877.938 \\
\text { (Valor p: } \\
0,000)\end{array}$ & 1244.178 (Valor p: 0,00 & & 1436.939 (valor $\mathrm{p}=0.000$ & \\
\hline \multicolumn{6}{|c|}{ Método de extracción: Análisis de componentes principales. Método de rotación: } \\
\hline $\begin{array}{l}\text { a. La rotación ha co } \\
\text { iteraciones. }\end{array}$ & vergido en 5 & $\begin{array}{l}\text { a. La rotación ha conv } \\
\text { iteraciones. }\end{array}$ & ergido en 3 & $\begin{array}{l}\text { a. La rotación ha conver } \\
\text { iteraciones. }\end{array}$ & ergido e \\
\hline
\end{tabular}

Fuente: Elaboración propia 
Respecto a la escala NEP, con base a la recomendación de los autores y para comprobar la dimensionalidad, se llevó a cabo un análisis factorial sin rotación, con el fin de demostrar que los ítems se agrupan en un solo factor. El primer factor cuenta con un 20 por ciento de varianza total extraída, lo cual sugiere que no se puede considerar como unidimensional, comparado con el 31.3 por ciento de los autores. Para analizar la multidimensionalidad de la escala, se realizaron análisis factoriales con rotación varimax, los resultados muestran que las variables no se correlacionan entre sí muy bien, ya que el KMO (0.679) es relativamente bajo. El análisis extrajo 4 de los 5 factores de Dunlap et al., (2000): F1 la realidad de los límites del crecimiento (4 ítems); F2 posibilidad de una ecocrisis (3 ítems); F3 rechazo a la excepción (2 ítems) y F4 la fragilidad del balance de la naturaleza ( 2 ítems) y la faceta de anti-antropocentrismo se elimina. Estos factores explican una varianza total extraída de 65.5 por ciento considerándose aceptable (Tabla 7). Por lo tanto, se encuentran diferencias en la estructura de creencias ambientales respecto a los resultados obtenidos por Dunlap et al., (2000): F1 la realidad de los límites del crecimiento (3 ítems); F2 posibilidad de una ecocrisis (3 ítems); F3 rechazo a la excepción (3 ítems) y F4 la fragilidad del balance de la naturaleza (3 ítems) y anti-antropocentrismo (3 items).

\section{Análisis clúster}

Tras este resultado, las puntuaciones factoriales obtenidas para cada individuo se utilizaron en una segunda fase como bases para segmentar por el análisis clúster o de conglomerados. El número de segmentos fue determinado por medio del análisis de clúster jerárquico, de tal forma que se identificaron 2 segmentos con diferencias significativas dentro de los 13 factores analizados $(\mathrm{p}<0.05)$ con los siguientes tamaños: grupo 1,319 individuos que representa el 63 por ciento y el grupo 2, con 187 individuos que comprende el 37 por ciento del total de la muestra. El primer segmento se denomina participativo ambientalmente, se caracteriza por aquellas personas que tienen interés por el medio ambiente (compromiso afectivo), son parte activa ambiental (compromiso verbal) que fomentan la participación colectiva y creen en la fragilidad del balance de la naturaleza, aunque consideran que el equilibrio de la naturaleza es lo suficientemente fuerte para hacer frente a los impactos de las naciones industriales modernas y no les preocupa la contaminación acústica, por ello, en este grupo no se reflejan los valores ambientales. 
Tabla 8. Análisis Clúster.

\begin{tabular}{llll} 
& Segmento 1 & Segmento 2 & Sig. \\
\hline Actitudes & $63 \%$ & $37 \%$ & \\
\hline Compromiso intencional & -0.343 & 0.58511 & .000 \\
\hline Interés por el medio ambiente & 0.10447 & -0.17821 & .002 \\
\hline Frustración por las acciones de organizaciones & -0.09774 & 0.16674 & .004 \\
\hline Compromiso de colaboración ambiental & & 0.13414 & .021 \\
\hline Preocupación por la contaminación & -0.07863 & 0.53435 & .000 \\
\hline Participación activa ambiental & -0.31324 & -0.33465 & .000 \\
\hline Cambio en estilos de vida & 0.19617 & 0.25119 & .000 \\
\hline Valores & -0.14725 & & .000 \\
\hline Conservación ambiental & & 0.2345 & .000 \\
\hline Respeto al medio ambiente & -0.38398 & 0.65502 & .000 \\
\hline Creencias & -0.26218 & 0.44725 & .000 \\
\hline La realidad de los límites del crecimiento & & & .000 \\
\hline Posibilidad de una ecocrisis & -0.16551 & 0.28234 & .000 \\
\hline Rechazo a la excepción & 0.13747 & 0.79512 & \\
\hline La fragilidad del balance de la naturaleza & & 0.36554 & \\
\hline Fute: Elat & & & \\
\hline
\end{tabular}

Fuente: Elaboración propia

El segundo segmento, se llama consistente ambientalmente, la mayoría de los factores se concentran en este grupo con valores positivos, se caracteriza por individuos que responden de manera estable y confiable frente a los estímulos ambientales, se frustran por las acciones negativas (compromiso afectivo) de organizaciones (industria y gobierno), tienen un compromiso (verbal) de colaboración ambiental, les preocupa la contaminación tanto en alimentos como en el aire (compromiso afectivo), y están cambiando los estilos de vida (compromiso verbal), le dan importancia a los valores de conservación y respeto por el medio ambiente, creen que reside una realidad sobre los límites del crecimiento, le dan mucha importancia a la posibilidad de una ecocrisis, sin embargo consideran que los seres humanos están aprendiendo de cómo funciona la naturaleza para controlarla (rechazo a la excepción) (Tabla 8).

De forma adicional, se analizaron los segmentos con las variables sociodemográficas edad y nivel de ingresos, para contrastar si existe asociación se realizó la prueba de chi-cuadrado 
encontrándose relaciones significativas. Ambos segmentos presentan diferencias significativas (Tabla 9), por ejemplo, en el segmento participativo ambientalmente la edad se concentra en 36-44 años (78 por ciento) y de 55 a más de 65 años, tienen ingresos medios ( 76 por ciento). En el caso del segmento consistente ambientalmente, tienen edad entre 45-54 años (67 por ciento) y sus niveles de ingresos van de medio alto ( 29 por ciento) a alto ( 6 por ciento).

Tabla 9. Segmentos por edad y nivel de ingresos.

\begin{tabular}{|c|c|c|c|}
\hline \multirow[t]{2}{*}{ Variables } & Participativo ambientalmente & $\begin{array}{l}\text { Consistente } \\
\text { ambientalmente }\end{array}$ & \multirow[t]{2}{*}{ Test X2 } \\
\hline & $63 \%$ & $37 \%$ & \\
\hline \multicolumn{3}{|l|}{ Edad } & \multirow{7}{*}{0.000} \\
\hline 18-24 años & $60 \%$ & $40 \%$ & \\
\hline 25-35 años & $61 \%$ & $39 \%$ & \\
\hline 36-44 años & $78 \%$ & $22 \%$ & \\
\hline 45-54 años & $33 \%$ & $67 \%$ & \\
\hline 55-64 años & $100 \%$ & $0 \%$ & \\
\hline 65 o más años & $100 \%$ & $0 \%$ & \\
\hline \multicolumn{3}{|c|}{ Nivel de ingresos } & \multirow{6}{*}{0.000} \\
\hline Alto & $0 \%$ & $6 \%$ & \\
\hline Medio alto & $14 \%$ & $29 \%$ & \\
\hline Medio & $76 \%$ & $53 \%$ & \\
\hline Medio bajo & $7 \%$ & $12 \%$ & \\
\hline Bajo & $3 \%$ & $0 \%$ & \\
\hline
\end{tabular}

Fuente: Elaboración propia

\section{Conclusiones}

El estudio ha permitido conocer los segmentos de los consumidores según las variables de tipo psicográfico como las actitudes, valores y creencias ambientales. Las variables sociodemográficas se analizaron para tener un contexto de la zona estudiada, en el cual de cada 10 entrevistados, 7 han consumido productos orgánicos en los últimos 6 meses, comprobando la tendencia del crecimiento en el sector. Aunque Sonora no es uno de los principales estados con manejo de áreas orgánicas, el potencial es muy alto.

Desde esta perspectiva, la investigación aporta directrices para comprender y conocer el comportamiento ambiental de los consumidores, en este sentido, se advierte que los alimentos orgánicos que más consumen en la región son verduras y frutas, y en menor medida los cereales, lácteos, dulces y carnes, reflejando que las decisiones de compra se hace por aquellas opciones que perciben con un menor riesgo de residuos de pesticidas, es decir, cada vez se manifiesta 
mayor conocimiento sobre la composición de los productos alimentarios y sus efectos sobre el organismo (Contreras y Gracia, 2005).

Por otro lado, se identificaron dos segmentos (participativo ambientalmente y consistente ambientalmente), a diferencia de otros estudios realizados en el contexto mexicano que han encontrado cinco y cuatro grupos respectivamente (González et al. 2015; Escobar-López et al., 2017;), es posible que esto obedezca al criterio de segmentación desarrollado, en el primero, se utilizaron las variables de reducción, reuso, reciclaje y compra de productos ecológicos y luego se perfiló con base en variables demográficas, eficacia percibida y valores ambientales y sociales y, en el segundo, se basó en la Food Choice Questionnaire (FCHQ).

Profundizando más en el análisis, el segmento participativo ambientalmente es el grupo con más integrantes jóvenes, con ingresos medios y manifiestan actitudes ecológicas como colaborar con trabajos de reciclaje, limpieza en las calles entre otras actividades positivas (compromiso verbal y afectivo) al medio ambiente (Chan, 2001), sin embargo, no son consistentes en sus acciones (carecen de un compromiso real), por ello los valores ambientales no están reconocidos y sus creencias se basan en que el problema ambiental no es tan grave. Así que en el momento de la compra pueden surgir dilemas éticos que hacen difícil determinar lo que "esta correcto" y lo que "esta incorrecto" (Leão de Carvalho et al., 2015), de tal forma, que los esfuerzos de marketing de las organizaciones deben ser mayores para lograr incentivar la compra de productos orgánicos.

En el caso del segmento consistente ambientalmente, los consumidores son conscientes del impacto que tienen sus acciones en el medio ambiente, en los problemas sociales y de salud de su localidad, son jóvenes adultos y adultos, pero en una proporción menor, ya que es un grupo más reducido. Estos resultados son coincidentes con los estudios de Díaz Víquez et al. (2015); González et al. (2015) y Escobar-López et al., (2017); que muestran que el rango de edad de 36-54 años, son los consumidores potenciales, debido a que son considerados económicamente activos, lo que a su vez se ve expresado en los niveles de ingresos de medio a alto. Además, cuentan con valores que pueden transformar su comportamiento más positivamente, tal situación es una oportunidad para ejercer una influencia en la recepción y percepción de mensajes publicitarios por parte de los productores de alimentos orgánicos como lo sugiere Watson et al., (2002), principalmente para aquellos productos procesados que son los de menor consumo. Este grupo tiene fuertes creencias sobre el impacto del crecimiento poblacional y una ecocrisis.

Por el contrario, la fase de anti-antropocentrismo no es identificada entre los segmentos del área estudiada, consistente con el resultado de González et al. (2015) lo cual sugiere que se tiene una perspectiva o visión enfocada en el derecho del hombre sobre la naturaleza y los seres vivos, es decir que las personas entrevistadas no consideran que los seres humanos y animales/ naturaleza tengan derechos equivalentes, ellos se centran más en aspectos que tienen que ver con el crecimiento, ecocrisis, excepción y el balance de la naturaleza, descubrimientos similares a los de Bechtel et al., (1999). Este hallazgo presenta oportunidades y retos para las organizaciones privados y públicos, las primeras, permitirán desarrollar sus estrategias de marketing sobre los alimentos orgánicos de la zona considerando estos segmentos básicamente para los supermercados, donde los productos orgánicos pueden ser adquiridos como una opción saludable, practica y que ahorre tiempo a los consumidores y las segundas, incluir la educación ambiental en sus estímulos para mejorar la concientización de las personas, resaltando la influencia de su consumo sobre las condiciones ecológicas del planeta (Vanhonacker et al., 2013). 
Como limitaciones de la investigación, al validar las escalas se deben incorporar instrumentos de medida que han sido desarrollados y testados en otros países y en otras culturas (Grande y Abascal, 2014), en el caso de la escala dunlap, al igual que los estudios de Corral et al., (1997) y Vozmediano y San Juan (2005), se ha encontrado que varía según las poblaciones, no existe acuerdo sobre su naturaleza factorial y, por tanto, se puede modificar la estructura de las creencias ambientales. En este sentido, Dunlap et al., (2000) manifiestan que la decisión de utilizar la escala como variable única o como conjunto de dimensiones debe basarse en los resultados obtenidos en cada trabajo particular. Por lo tanto, como futuras líneas de investigación, se pueden analizar otros escenarios con similitudes como países de Latinoamérica, para conocer sus actitudes, valores y creencias ambientales y si existen diferencias entre ellos. Asimismo, se podría seguir ahondando en relacionar más variables con las conductas ambientales como escalas que midan la conciencia de la salud y percepción de los consumidores sobre la seguridad alimentaria.

\section{Referencias}

Aguilar Salinas, W. E., Ojeda Benitez, S., \& Castro Rodríguez, J.R. (2012). Perfil sociodemográfico de los consumidores asociados a un comportamiento ambiental. Puente Revista Científica, 6 (2), 81-86. http://dx.doi.org/10.18566/ puente.v6n2.a0\&\#13;9.

Ahmed, M. I., Kamalanabhan, T. J., \& Chih, P. L. G., (2001). Green marketing and its implications on consumers and businesses in Malaysia: An empirical study. Journal of Human Ecology, 12 (4), 245-249. https://doi.org/10.1080/ 09709274.2001 .11907611

Alba, J. W., \& Hutchinson, J. W. (1987). Dimensions of consumer expertise, Journal of Consumer Research, 13, 41154. https://doi.org/10.1086/209080

Bagozzi, R.P., Baumgartner, H., \& Yi, Y. (1992). State versus action orientation and the theory of reasoned action: an application to coupon usage. Journal of Consumer Research, 18 (4), 505-18. https://doi.org/10.1086/209277

Balderjahn, I., Buerke, A., Kirchgeorg, M., Peyer, M., Seegebarth, B., \& Wiedmann K. -P. (2013). Consciousness for sustainable consumption: scale development and new insights in the economic dimension of consumers' sustainability. Academy of Marketing Science Review 3(4),181-192. Doi 10.1007/s13162-013-0057-6

Beatty, S., Kahle, L., Homer, P., \& Misra, S. (1985). Alternative measurement approaches to consumer values: the list of values and the Rokeach value survey. Psychology and Marketing 2, 181-200. https://doi.org/10.1002/ mar.4220020305

Bechtel R. B., Corral, V., \& Pinheiro J.D.Q. (1999). Environmental belief systems United States, Brazil, and Mexico. Journal of Cross-Cultural Psychology, 30 (1), 122-128. https://doi.org/10.1177/0022022199030001008

Bhatia M., \& Jain A. (2013). Green Marketing: A Study of Consumer Perception and Preferences in India. Electronic Green Journal, 1, 36. Disponible en: https://escholarship.org/uc/item/5mc39217 y consultado: 12/06/2015.

Bratt, C. (1999). Consumers' environmental behavior: generalized, sector-based, or compensatory?. Environment \& Behavior, 29, 515-531. https://doi.org/10.1177/00139169921971985

Brundtland, G. H. (1987). Report of the World Commission on Environment and Development: Our Common Future. United Nations. Disponible en: http://www.un-documents.net/our-common-future.pdf y consultado: 23/07/2014.

Carrete L., Castaño R., Felix R., Centeno, E., González, E. (2012). Green consumer behavior in an emerging economy: confusion, credibility, and compatibility. Journal of Consumer Marketing, 29 (7). 470 - 481 . http://dx.doi. org/10.1108/07363761211274983

Catlin, J. R., \& Wang Y. (2013). Recycling gone bad: When the option to recycle increases resource consumption. Journal of Consumer Psychology, 23 (1), 122-127. https://doi.org/10.1016/j.jcps.2012.04.001 
Catton, W. R., \& Dunlap R. E., (1978). Environmental sociology: A new paradigm. The American Sociologist, 13, 4149. Disponible en: https://fenix.tecnico.ulisboa.pt/downloadFile/3779580063239/Dunlap--NEW-PARADIGM.. pdf y consultado: 11/07/2013.

Chan, K. (1999). Market segmentation of green consumers in Hong Kong. Journal of International Consumer Marketing, 12 (2), 7-24. https://doi.org/10.1300/j046v12n02_02

Chan, R.Y.K. (2001). Determinants of Chinese' Consumers' Green Purchase Behavior. Psychology and Marketing, 18 (4), 389-413. https://doi.org/10.1002/mar.1013

Contreras, J., \& Gracia, M. (2005) Alimentación y cultura, perspectivas antropológicas, Ed. Ariel, Barcelona, España. Corral-Verdugo V. (1996). A structural model of reuse and recycling in Mexico. Environment \& Behavior 28, 665-696. https://doi.org/10.1177/001391659602800505

Corral-Verdugo, V., R. B., Bechtel, L. I., Armendáriz \& Esquer A. N., (1997). La estructura de las creencias ambientales en universitarios mexicanos: El Nuevo Paradigma Ambiental. Revista Mexicana de Psicología. 14(2), 173- 181.

Corral-Verdugo V. \& Armendáriz L.I., (2000). The "New Environmental Paradigm" in a Mexican Community. The Journal of Environmental Education. 31 (3), 25-31. https://doi.org/10.1080/00958960009598642

Corral-Verdugo V., Hess S, Hernández B. \& Suarez E. (2009). Los fundamentos y la estructura de la acción proecológica, medidos en una escala de conductas protectoras del ambiente. pp. 71-88. En: Víctor Corral Verdugo (coord.) Conductas protectoras del ambiente, teoría, investigación y estrategias de intervención. Universidad de Sonora, Plaza y Valdés. México.

Crane, A. (2000). Facing the backlash: green marketing and strategic reorientation in the 1990's. Journal of Strategic Marketing, 8, 277-296. https://doi.org/10.1080/09652540050110011

Del Barrio, S., \& Luque, T. (2000). Análisis de ecuaciones estructurales, Técnicas de Análisis de Datos en Investigación de Mercados. Madrid: Pirámide.

Diamantopoulos, A., Schlegelmilch, B. B., Sinkovics, R. R., \& Bohlen G. M., (2003). Can socio-demographics still play a role in profiling green consumers? A review of the evidence and an empirical investigation. Journal of Business Research, 56, 465-480. https://doi.org/10.1016/s0148-2963(01)00241-7

Díaz Víquez, A., Pérez Hernández, A. \& Hernández Ávila, J. (2015). Caracterización del consumidor de productos orgánicos en la ciudad de Toluca. Revista Mexicana de Agronegocios. 19 (36), 1178-1187. Disponible en: https:// www.redalyc.org/pdf/141/14132408004.pdf y consultado: 07/12/2016.

Dietz T., Stern P.C. \& Guagnano G.A. (1998). Social structural and social psychological bases of environmental concern. Environment \& Behavior 30 (4), 450-471. https://doi.org/10.1177/001391659803000402

Dunlap, R. E., \& Van Liere, K. D. (1978). The "new environmental paradigm:” A proposed measuring instrument and preliminary results. Journal of Environmental Education, 9, 10-18. http://dx.doi.org/10.1080/00958964.1978.108 01875

Dunlap, R. E., K. D., Van Liere, Mertig, A. G., \& Jones, R. E. (2000) New Trends in Measuring Endorsement of the New Ecological Paradigm: A Revised NEP Scale. Journal of Social Issues, 56 (3) 425-442. https://doi. org/10.1111/0022-4537.00176

Elkington, J. (1997). Cannibals with forks: The triple bottom line of 21st century business. London: Capstone Publication.

Ellen, P.S., (1994). Do we know what we need to know - objective and subjective knowledge effects on pro- ecological behaviors. Journal of Business Research, 30 (1), 43-52. https://doi.org/10.1016/0148-2963(94)90067-1

Escobar-López, S.Y., Espinoza-Ortega, A. Vizcarra-Bordi, I., Thomé-Ortiz, H. (2017). The consumer of food products in organic markets of central Mexico. British Food Journal. 119 (3), 558-574. https://doi.org/10.1108/BFJ-072016-0321

FIBL (2017). FIBL Survey 2017. Global market, Organic monitor 2017. Disponible en: http://www.fibl.org/de/startseite.html Consultado: 03/02/2017.

Fraj, A. E. (2003). Las variantes demográficas y socioeconómicas como determinantes del comportamiento de reciclaje: Su importancia sobre la gestión de residuos sólidos. Revista de gestión pública y privada, 8, 103-118.

Fraj, E., Martínez, E., \& Grande, I. (2004). Un estudio exploratorio sobre las variables psicográficas que influyen en el comportamiento del consumidor ecológico. Revista de Economía y Empresa, 50 (21), 61-87. 
Fraj, E. \& Martínez, E. (2005). El nivel de conocimiento medioambiental como factor moderador de la relación entre la actitud y el comportamiento ecológico. Investigaciones Europeas de Dirección y Economía de la Empresa, 11 (1), 223-243. Disponible en: http://redaedem.org/articulos/iedee/v11/111223.pdf

González, A. M. (2000). Los Valores Personales en el Comportamiento del Consumidor. Revisión de Diversas Metodologías Aplicadas al Marketing. ESIC Market, septiembre-diciembre (107), 9-36. Disponible en: https://www.esic. edu/documentos/revistas/esicmk/060130_479761_E.pdf

González, E. M., Felix, R., Carrete, L. Centeno R. \& Castaño R. (2015) Green Shades: A Segmentation Approach Based on Ecological Consumer Behavior in an Emerging Economy. Journal of Marketing Theory and Practice, 23(3), 287-302 http://dx.doi.org/10.1080/10696679.2015.1032395

Grande, I., \& Abascal, E. (2014). Fundamentos y técnicas de investigación comercial. (12 ed.) España: Esic.

Grubor A. \& Nenad D., (2016). Organic food consumer profile in the Republic of Serbia. British Food Journal. 118 (1), 164-182. http://dx.doi.org/10.1108/BFJ-06-2015-0225

Gutiérrez, C., Tuñón, E., Limón, F., Morales, H., \& Nigh, R. (2012). Representaciones sociales de los alimentos orgánicos entre consumidores de Chiapas. Estudios sociales, 20 (39), 99-130.

Hair, J., Tatham, R., Anderson, R., \& Black, W. (2005). Análisis multivariante, Madrid: Pearson Prentice Hall.

Haws, K. L., Winterich K. P., \& Naylor R. W. (2014). Seeing the world through GREEN-tinted glasses: motivated reasoning and consumer response to environmentally friendly products. Journal of Consumer Psychology 24, 3 (2014) 336-354. http://dx.doi.org/10.1016/j.jcps.2013.11.002

Heberlein, T. A. (1972). The land ethic realized: some social psychological explanations for changing environmental attitudes. Journal of Social Issues, 28 (4), 79-87. https://doi.org/10.1111/j.1540-4560.1972.tb00047.x

Holt, D. (1997). Poststructuralist lifestyle analysis Conceptualizing the Social Patterning of Consumption in Postmodernity. Journal of Consumer Research. 23 (4), 326-350 https://doi.org/10.1086/209487

Hsu, S.Y., Chang, C.C., \& Lin, T. T. (2016). An analysis of purchase intentions toward organic food on health consciousness and food safety with/under structural equation modeling. British Food Journal, 118 (1), 200-216. https://doi.org/10.1108/bfj-11-2014-0376

IFOAM (2016). The world of organic agriculture. Statistics \& emerging trends 2016. Research Institute of Organic Agriculture. Disponible en: https://www.organic-world net/yearbook/yearbook-2016.html y consultado: 21/04/2016.

Ip, Y. K. (2003). The marketability of eco-products in China's affluent cities: a case study related to the use of insecticide. Management of Environmental Quality, 14(5), 577-589. https://doi.org/10.1108/14777830310495731

Instituto Interamericano de Cooperación para la Agricultura IICA (2016) Agricultura orgánica en ALC crece en producción y exportación. Disponible en: http://www.iica.int/es/prensa/noticias/agricultura-orgánica-en-alc-crece-en-producción-y-exportación y consultado: 27/09/2016.

Kahle, L., \& S., Goff. (1983). A Theory and Method for Studying Values En: Kahle, L. (Ed.): Social Values and Social Change: Adaptation to Life in America, N.Y.: Praeger: 324.

Kahle, L., Gregory, R., \& Shoham, A. (2000). Findings of LOV throughout the world, and other evidence of Cross-national consumer psychographics: Introduction En L. Kahle (Ed.): Cross-national consumer Psychographic. New York: International Business Press. 1-13.

Kim, H. Y., \& Chung, J. E. (2011). Consumer purchase intention for organic personal care products. Journal of Consumer Marketing. 28 (1), 40-47. https://doi.org/10.1108/07363761111101930

Kim, Y. (2011). Understanding green purchase:The influence of collectivism, personal values and environmental attitudes, and the moderating effect of perceived consumer effectiveness. Seoul Journal of Business, 17 (1), 65-92. http://s-space.snu.ac.kr/bitstream/10371/75584/1/05_Yeonshin\%20Kim.pdf

Kotchen, M. J., \& Reiling, S.D. (2000). Environmental attitudes, motivations and contingent valuation of nonuse values: a case of study involving endangered species. Ecological Economics, 32, 93-107. https://doi.org/10.1016/ s0921-8009(99)00069-5

Leão de Carvalho, B., Salgueiro M de F., Rita P. (2015). Consumer Sustainability Consciousness: A five dimensional construct. Ecological Indicators 58 (2015) 402-410. https://doi.org/10.1016/j.ecolind.2015.05.053

Laroche, M., Bergeron, J. \& Barbaro-Forleo, G. (2001). Targeting consumers who are willing to pay more for environmentally friendly products. Journal of Consumer Marketing. 18 (6), 503-520. https://doi.org/10.1108/ eum0000000006155 
Lee YJ, de Young R. \& Marans R.W. (1995). Factors influencing individual recycling behavior in office settings. Environment \& Behavior. 27, 380-403. https://doi.org/10.1177/0013916595273006

Maloney, M. P. \& Ward, M. P. (1973). Ecology-let's hear from people -objective scale for measurement of ecological attitudes and knowledge. American Psychologist. 28 (7), 583-586. https://doi.org/10.1037/h0034936

Maloney, M. P., Ward, M. P., \& Braucht, G. N. (1975). A revised scale for the measurement of ecological attitudes and knowledge. American Psychologist. 30, 787-790. https://doi.org/10.1037/h0084394

McCarty, J. A., \& Shrum, L. J. (1994). The recycling of solid wastes: personal values, value orientations, and attitudes about recycling as antecedents of recycling behavior. Journal of Business Research, 30 (1), 53-62. https://doi. org/10.1016/0148-2963(94)90068-X

Mesías Díaz, F. J., Martínez-Carrasco P. F., Martínez Paz, J. M., \& Gaspar García, P., (2012). Consumer knowledge, consumption, and willingness to pay for organic tomatoes. British Food Journal. 114 (3), 318 - 334. http://dx.doi. org/10.1108/00070701211213447

Michaelidou, N., \& Hassan, L. M. (2008). The role of health consciousness, food safety concern and ethical identity on attitudes and intentions towards organic food. International Journal of Consumers Studies. 32 (2), 163 170. https://doi.org/10.1111/j.1470-6431.2007.00619.x

Miquel, S., Bigné E., Levy J.P., Cuenca A., \& Miquel, M. J. (1996). Investigación de Mercados, McGraw-Hill Interamericana.

Nair, A., \& Berthon, P. (2003) Creating the Customer: The Influence of Advertising on Consumer Market Segments - Evidence and Ethics. Journal of Business Ethics 42: 83-99, https://link.springer.com/content/pdf/10.1023\%2FA\%3A1021620825950.pdf

National Gegraphic (2014). Greendex Mexicans. Disponible en: http://images.nationalgeographic.com/wpf/media-live/file/Greendex-Mexicans_FINAL-cb1409256142.pdf y consultado 12/10/2014.

Nisbet, E. K., Zelenski, J. M. \& Murphy S. A. (2009). Nature relatedness scale: linking individuals' connection with nature to environmental concern and behavior. Environment and Behavior. 41 (5), 715-740. https://doi. org/10.1177/0013916508318748

Orduña, V., Espinoza, N. \& González, D. (2009). Relación entre variables demográficas, variables contextuales, conocimiento ambiental y el ahorro de agua En: Víctor Corral Verdugo (coord.) Conductas protectoras del ambiente, teoría, investigación y estrategias de intervención. Hermosillo: Universidad de Sonora, Plaza y Valdés.

Peattie, K (2001). Towards Sustainability: The Third Age of Green Marketing. The Marketing Review. 2 (2), 129-146. https://doi.org/10.1362/1469347012569869

Pedret, R., Sagnier, L. \& Camp, F. (2003). Herramientas para segmentar mercados y posicionar productos, análisis de información cuantitativa en investigación comercial. Madrid: Deusto.

Pino, G., Peluso, A. M. \& Guido, G. (2012). Determinants of regular and occasional consumers' intentions to buy organic food. The Journal of Consumer Affairs. 46 (1), 157-169. https://doi.org/10.1111/j.1745-6606.2012.01223.x

Placet, M., Anderson, R., \& Fowler, K. M. (2005). Strategies for sustainability. Res. Tech- nol. Manag. 48 (5), $32-41$. https://doi.org/10.1080/08956308.2005.11657336

Ramsey, C. \& Rickson R. (1976). Environmental knowledge and attitudes. Journal of Environmental Education. 8, 10-18. https://doi.org/10.1080/00958964.1976.9941552

Ramírez M. (2016). El mercado de productos orgánicos crece un 10\% cada año. Negocios. Marzo. Disponible en: http://www.milenio.com/negocios/productos_organicos-veganos-vegetarianos-comer_sano_0_708529215.html y consultado 01/04/2016.

Rivas, J., Grande, I. (2004). Comportamiento del Consumidor, Madrid, Esic.

Roberts, J. A. (1996). Green consumers in the 1990S: Profile and implications for advertising. Journal of Business Research. 36 (3), 217-231. https://doi.org/10.1016/0148-2963(95)00150-6

Rokeach, M. (1973). The Nature of Human Values. New York: The Free Press.

Rodríguez, S., Camarero C. \& Gutiérrez J. (2002). Lealtad y valor en la relación del consumidor. Una aplicación al caso de los servicios financieros. XIV Encuentro de Profesores Universitarios de Marketing. Granada, España.

Romero Valenzuela, D. M., Camarena Gómez B. O. \& Camarena Gómez, D. M. J. (2016). Oferta de productos hortofrutícolas orgánicos en Hermosillo, Sonora. Vertice Universitario 69, 2-9. 
Rose, G. \& Shoham A. (2000). The Values of American and Japanese Mothers: An Application of LOV in the U.S. and Japan. Journal of Euromarketing. 8, 1-2. http://dx.doi.org/10.1300/J037v08n01_04

SAGARPA (2013). Fomenta SAGARPA producción y consume de alimentos orgánicos y el mundo. Disponible en: https://www.gob.mx/aserca/prensa/fomenta-sagarpa-produccion-y-consumo-de-alimentos-organicos-en-mexico-y-el-mundo y consultado: 03/05/2013.

SAGARPA (2016). Avanza México en la producción de alimentos orgánicos certificados. Disponible en: https://www. gob.mx/aserca/prensa/avanza-mexico-en-la-produccion-de-alimentos-organicos-certificados-41243?idiom=es y consultado: 15/07/2016.

SAGARPA, SIAP (2016) Atlas agroalimentario 2016. Disponible en: https://nube.siap.gob.mx/gobmx_publicaciones_siap/pag/2016/Atlas-Agroalimentario-2016 y consultado: 23/09/2016.

SAGARPA, SIAP (2017) Atlas agroalimentario 2017. Disponible en: http://online.pubhtml5.com/clsi/ibhs/\#p=118 y consultado: 03/02/2017.

Salgado-Beltrán, L., Gil, A., Subira, E. \& Beltrán-Morales, L.F. (2006) Teoría de efectos olvidados en el consumo sustentable de productos ecológicos. Pp. 223-239. En: Libro Luis F. Beltrán-Morales, José Urciaga-García \& Alfredo Ortega-Rubio (Eds). Desarrollo Sustentable: Mito o Realidad? CIBNOR, SC., CONACYT, SEMARNAT, INE, México.

Salgado-Beltrán L., Bravo Díaz B. (2015). Consumo verde en el norte de Sonora. Revista Agroproductividad. Año 8.8 (4), 42-47. http://132.248.9.34/hevila/Agroproductividad/2015/vol8/no4/7.pdf

Salgado-Beltrán L., Beltrán-Morales, L.F. (2011). Factores que influyen en el consumo sustentable de productos orgánicos en El noroeste de México. Revista Ecosistemas y Recursos Agropecuarios. 27 (3), 265-279. http://dx.doi. org/10.19136/era.a27n3.105

Schlegelmilch, B. B., A. Diamantopoulos \& Bohlen G. M., (1994). The Value of Sociodemographic Characteristics for Predicting Environmental Consciousness" en: Park, C. W. Smith, D. C. (edits.): Marketing Theory and Applications: The Proceedings of the 1994 American Marketing Association's Winter Educator's Conference. 5, AMA, Chicago, IL, 348-349.

Schwartz, S. H. (1970). Moral decision making and behavior en: J. Macauley \& L. Berkowitz (Eds.), Altruism and helping behavior. New York: Academic Press.

Schwartz, S. H. (1977). Normative influences on altruism. Advances in experimental social psychology, 10, 221-279. https://doi.org/10.1016/s0065-2601(08)60358-5

Schwartz, S. H. (1994). Are there universal aspects in the structure and contents of human values? Journal of Social Issues. 50 (4), 19-45. https://doi.org/10.1111/j.1540-4560.1994.tb01196.x

Shiffman, L. G. \& Lazar, L. (2010). Comportamiento del consumidor, Ed. Prentice Hall, 10ma, edición, México.

Smith, P. B. \& Schwartz, S. H. (1997). Values En: Berry J.W., Segall M.H. \& C. Kagitibasi (Eds.) Handbook of Cross-Cultural Psychology, 3, Social Behavior and Applications. 77-118. Needham Heights, MA: Allyn \& Bacon.

Stern, P. C., Dietz, T. \& Kalof, L. (1993). Value orientations, gender, and environmental concern. Environment and Behavior. 25 (3), 322-348. http://dx.doi.org/10.1177/0013916593255002

Stern, P. C. (2000). Toward a coherent theory of environmentally significant behavior. Journal of Social Issues. 56, 407-424. http://dx.doi.org/10.1111/0022-4537.00175

Thøgersen J., Ölander F. (2002) Human values and the emergence of sustainable consumption pattern: a panel study. Journal of Economic Psychology, 23 (5), 605-630. http://dx.doi.org/10.1016/S0167-4870(02)00120-4

Tracy, A. P. \& Oskamp, S. (1984). Relationships among ecologically responsible behaviors. Journal of Environmental Systems. 13, 115-126. https://doi.org/10.2190/6tn8-48wt-63xt-chen

Vanhonacker, F., Van Loo, E. J., Gellynck, X., Verbeke, W. (2013). Flemish consumer attitudes towards more sustainable food choices. Appetite. 62, 7-16. http://dx.doi.org/10.1016/j.ecolind.2015.05.053

Vining, J. \& Ebreo, A. (1990). What makes a recycler? A comparison of recyclers and nonrecyclers. Environmental Behavior. 22, 55-73. https://doi.org/10.1177/0013916590221003

Vozmediano, L., \& San Juan, C. (2005). Escala Nuevo Paradigma Ecológico: propiedades psicométricas con una muestra española obtenida a través de Internet. Medio Ambiente y Comportamiento Humano. 6 (1), 37-49. Disponible en: https://mach.webs.ull.es/PDFS/Vol6_1/VOL_6_1_d.pdf 
Ward, J. H. (1963). Hierarchical grouping to optimize an objective function. Journal of the American Statistical Association. 58, 236-44. Disponible en: http://iv.slis.indiana.edu/sw/data/ward.pdf

Watson, J.J., Guillan, T., Lysonski S. \& Raymore L. (2002). Cultural values and important possessions: A cross-cultural analysis. Journal of Business Research. 55 (11), 923-931. https://doi.org/10.1016/s0148-2963(01)00212-0

Williams, R. M. (1979). Change and stability in values and value perspectives: A sociological perspective En: Rokeach, M. (Ed.): Understanding human values: individual and societal, N.Y.: Free Press. https://doi.org/10.1093/ $\mathrm{sf} / 59.4 .1330$

Wier, M. \& Calverley, C. (2002). Market potential for organic foods in Europe. British Food Journal. 104 (1): 45-62. http://dx.doi.org/10.1108/00070700210418749

Yankelovich, D. \& Smith, G. (1981). New rules: searching for self-fulfillment in a world turned upside down. Foreign Affairs. 60 (1), 206. https://doi.org/10.2307/20041029 